\title{
La gouvernance démocratique du secteur de la sécurité et la réforme de l'armée au Nigéria
}

\author{
E. Remi Aiyede \\ Maître de conférences, Département de science politique, \\ University of Ibadan, Nigeria
}

\begin{abstract}
Introduction
Le Nigéria a entrepris de réformer son secteur de la sécurité au début du nouveau millénaire alors qu'il était en phase de transition d'un régime militaire vers un régime civil. Pour commencer, il a fallu convaincre les forces armées de réintégrer leurs casernes et se cantonner à leur rôle de gardiens de l'Etat comme il est prévu dans la constitution. Des élections ont eu lieu, et un régime civil a pris le contrôle du gouvernement ainsi que de l'armée dans le cadre d'un bref programme de transition organisé par le régime du général Abdulsalami Abubakar. Le gouvernement Obasanjo (1999-2007) a mis en œuvre une série de réformes en vue de renforcer les institutions politiques après des années de tyrannie et de crise économique. La Stratégie nationale pour l'autonomisation et le développement économiques sarticulait autour de quatre thèmes principaux: améliorer l'environnement macroéconomique, poursuivre des réformes de fond, rationaliser la gestion des dépenses publiques et mettre en œuvre des réformes juridiques et institutionnelles. Le document Vision 20: 2020 est devenu le plan directeur encadrant les efforts du Nigéria pour figurer parmi les vingt économies les plus performantes et concurrentielles au monde (NNPC 2009).
\end{abstract}

Bien que ces documents ne mentionnent pas la réforme du secteur de la sécurité de manière explicite, le gouvernement Obasanjo a sollicité l'aide de

Comment citer ce chapitre du livre:

Aiyede, E. R. 2015. La gouvernance démocratique du secteur de la sécurité et la réforme de l'armée au Nigéria. Dans: Bryden, A et Chappuis, F (dir. publ.) Gouvernance du secteur de la Sécurité : Leçons des expériences ouest-africaines, Pp. 103-124. London: Ubiquity Press. DOI: http://dx.doi.org/10.5334/bav.f. Licence: CC-BY 4.0. 
la communauté internationale pour mettre en œuvre ses processus de réforme militaire. Au nombre des agences extérieures actives dans le domaine de la réforme des armées, l’on peut citer une société privée (Military Professional Resources Incorporated - MPRI), le Programme international du gouvernement américain pour l'éducation et la formation militaires (International Military Education and Training programme) et l'Equipe britannique de conseil sur la défense (British Defence Advisory Team). Alors que le Nigéria est toujours en proie à de graves problèmes sécuritaires, l'idée de réformer le secteur de la sécurité est restée à l'ordre du jour durant les divers gouvernements qui se sont succédé, particulièrement renforcée par l'impact dévastateur d'un régime militaire prolongé sur le moral des civils et même des militaires au lendemain de l'indépendance (Siollun 2013).

Les principales questions à traiter sont la prévention des coups d'Etat, la démilitarisation de la société, le contrôle de l'armée par les autorités civiles, le retrait des militaires de postes normalement réservés à la police, la nécessité de décentraliser les services de police, celle de renforcer la capacité de l'armée à lutter contre les insurrections, et enfin la réforme du système pénitentiaire et de justice pénale. Ces volets de la réforme du secteur de la sécurité sont devenus ces derniers temps les thèmes favoris des médias. Les efforts récemment déployés par l'armée pour lutter contre le terrorisme, notamment contre Boko Haram, ont placé ces questions au cœur des débats dans la société nigériane, principalement à cause des polémiques qui ont entouré l'action de l'armée contre Boko Haram (laquelle traîne en longueur et s'essouffle) et ont fait douter de sa capacité à être opérationnelle.

En 2014, le gouverneur Kashim Shettima a déclaré que les soldats nigérians étaient piètrement armés et peu motivés, accentuant ainsi l'urgence des débats (Onuoh 2014). D’autres sujets ont été évoqués: les déserteurs, les manifestations organisées par les femmes de soldats pour exiger que leurs maris soient mieux équipés, la corruption récurrente au sein de l'armée et le cas de plusieurs soldats ayant tiré sur leurs chefs pour avoir ordonné des missions trop dangereuses. Dans un contexte où un programme de réforme militaire est censé être en vigueur et où l'armée est de plus en plus sollicitée pour venir en aide aux autorités civiles (requérant un budget accru pour la défense), il est devenu impératif de s'impliquer activement à l'échelle du pays et même du monde (BBC 2015).

Qu'est-il advenu des efforts de réforme militaire au Nigéria? Dans quelle direction est-elle allée? S’est-elle arrêtée ou avait-elle pris un mauvais départ? Quelles sont les parties prenantes impliquées et quels rôles ont-elles joué? Comment la réforme peut-elle être ravivée et réorientée de façon à résoudre les problèmes sécuritaires de notre époque? La présente étude examine les réponses à ces questions et montre que le pays a perdu plusieurs occasions de faire de son armée une force efficace capable de repousser des attaques venues 
de l'extérieur et de garantir la sécurité intérieure dans le cadre d'un contrôle civil démocratique.

Au fond, la décision du général Obasanjo de modifier la constitution afin de prévoir un troisième mandat pour le président et les gouverneurs des Etats a ralenti l'action de son gouvernement en faveur d'une réforme militaire. Cela a permis à l'armée de s'approprier le contrôle de la réforme du secteur de la défense au détriment des autorités civiles. Ainsi, la réforme s'est trouvée influencée par les priorités des nouveaux chefs militaires et par leur incapacité à corriger la mauvaise gestion de l'armée due à la mise en avant d'intérêts personnels et aux fréquents changements parmi les dirigeants. Cette situation a ensuite empêché la mise en œuvre d'une transformation systématique de l'armée. D’autres facteurs encore ont eu un impact sur le processus de réforme, par exemple, la mauvaise santé suivie du décès du président Yar' Adua, qui a succédé au président Olusegun Obasanjo, et l'émergence de Boko Haram en tant que menace majeure pour la sécurité. Sous le régime du président Goodluck Jonathan, la lutte anti-terroriste contre Boko Haram n'a pas seulement changé le cours de la réforme de l'armée, elle a aussi révélé les limites et les insuffisances des efforts accomplis à ce jour et a souligné les rivalités existant entre les institutions, notamment avec la police. Les tentatives de réforme se sont soldées par un tel échec qu’en 2014 le ministre de la Défense, le sénateur Musiliu Obanikoro, a évoqué l'idée d'un nouveau départ en vue de réformer l'armée (Eghaghe 2014: 1).

La présente étude se propose de montrer comment le Nigéria a perdu plusieurs occasions de transformer son armée en une force efficace capable de repousser des attaques venues de l'extérieur et de garantir la sécurité intérieure dans le cadre d'un contrôle civil démocratique. Elle montre aussi comment l'évolution de la dynamique démocratique (par exemple, la décision du général Obasanjo de modifier la constitution de façon à prévoir un troisième mandat pour le président et les gouverneurs des Etats) a freiné les efforts du gouvernement pour mettre en œuvre une réforme de l'armée. Cela a permis à cette dernière de s'octroyer le contrôle de la réforme du secteur de la défense au détriment des autorités civiles, empêchant la mise en œuvre d'une transformation systématique de l'armée. Ce chapitre commence par présenter l'histoire de l'armée depuis l'époque coloniale, puis examine les différents efforts déployés au lendemain de l'indépendance pour en faire une institution stable dédiée à la défense et à la sécurité du pays. Il donne les grandes lignes de la réforme militaire menée sous Obasanjo de 1999 à 2007 lorsque le pays est revenu à un régime civil après vingt ans de dictatures militaires. Ensuite, il passe en revue le contre-programme de transformation institué par l'armée, son degré d’application et les raisons de l'état actuel de la réforme militaire. Pour finir, il suggère une feuille de route prenant en compte les critères à satisfaire (restés lettre morte jusqu'ici) afin que les changements s'effectuent de manière systématique. 


\section{Le contexte de la gouvernance du secteur de la sécurité au Nigéria}

Ancienne colonie britannique, le Nigéria a accédé à l'indépendance le $1^{\text {er }}$ octobre 1960. A partir de ce moment-là, une forme de gouvernement parlementaire a été mise en place, à savoir une fédération de trois régions (le Nord, l'Est et l'Ouest). En 1963, le pays est devenu une république. La même année, une quatrième région a été ajoutée, le Centre-Ouest.

Le Conseil de défense fédéral a été créé en 1957. Il comprend des représentants du gouvernement fédéral et des gouvernements régionaux et est présidé par le gouverneur général. C'est ce conseil qui a pris les toutes premières décisions officielles en matière de politique de défense et qui a défini lorganisation de larmée nigériane, y compris ses critères de recrutement et la nature des procédures parlementaires visant à établir et approuver le budget annuel. Il a pris la pleine responsabilité de l'armée juste après l'indépendance, terminant sa «nigérianisation » en 1960, bien qu'une grande proportion d'officiers britanniques ait continué de servir dans les rangs les plus élevés. A cette époque, la société civile et les médias étaient fortement impliqués dans la politique sécuritaire, comme on a pu le constater lors des manifestations qui ont accueilli la proposition du Pacte anglo-nigérian pour la défense et la participation continue du Nigéria aux opérations internationales de maintien de la paix au Congo après lexécution de Patrice Lumumba. Pendant ce temps, le Parlement a aidé à instaurer un quota dans le recrutement des officiers afin de refléter la diversité nationale (Alaga \& Akum 2013).

Cependant, les progrès accomplis en matière de réforme du secteur de la défense ont été stoppés net quand la Ie République a connu une fin abrupte le 15 janvier 1966 à la suite d'un coup d'Etat, conséquence ultime de la crise de gouvernance qui a marqué le bref régime de cette république. Les raisons principales étaient la confrontation officieuse entre le président et le premier ministre concernant les élections fédérales de 1964 et les élections truquées de 1965 dans l'Ouest qui ont abouti à l'effondrement de l'ordre public dans la région. Le coup d'Etat a été essentiellement imputé au peuple igbo car la presque totalité des hommes politiques et des dirigeants militaires de la Ie République assassinés venaient du Nord ou du Sud-Ouest, à l'exception d'un officier igbo.

Le pays est entré dans une guerre civile après un contre coup d'Etat le 29 juillet 1966 considéré comme un acte de vengeance car, à part le général Ironsi et Fajuyi, un seul parmi les dix officiers tués nétait pas d’origine igbo (Dudley 1973; Post \& Vickers 1973). Avec le contre coup d'Etat, l'esprit de cohésion engendré par un commandement militaire unifié a disparu; la hiérarchie militaire a été bouleversée lorsque le lieutenant-colonel Ojukwu a objecté à la promotion du lieutenant-colonel Gowon à la tête de l'Etat parce qu'il nétait pas le prochain candidat dans la chaîne de commandement en dessous du général Ironsi. Malgré les efforts déployés pour réconcilier les factions, le massacre des Igbos dans 
le Nord et la déclaration de l'Etat souverain du Biafra qui a suivi, ont annoncé le début d'une guerre civile qui allait durer de 1967 jusqu’en 1970. Ainsi, le 27 mai 1967, le conseil consultatif de la région de l'Est a mandaté Ojukwu pour promulguer l'avènement de la République indépendante du Biafra, déclaration suivie par celle de l'état d'urgence et la création par le général Yakubu Gowon d'une instance fédérale composée de 12 Etats chargée de gouverner le pays (Osaghae 1998; Panter-Brick 1970). Du fait de cette dynamique politique, les Igbos ont été mis à lécart de l'armée dans la période d’après-guerre.

Une autre conséquence de la guerre civile pour les militaires a été l'augmentation des effectifs de l'armée: de 7000 hommes environ organisés en seulement deux brigades d'infanterie en 1967, elle est passée à plus de 250000 officiers et soldats en 1970 (Bali 1989: 164). Ainsi, au lendemain des conflits, les efforts déployés pour réduire les effectifs et rendre l'armée plus agile sont devenus un thème central de la réforme et de la transition vers la démocratie. Malgré la promesse de démobilisation faite par le général Gowon, peu à été accompli à cet égard. Gowon a été renversé en 1975 pour ne pas avoir soutenu le programme de transition qui devait mettre fin au régime militaire en 1976. Le général Murtala Mohammed, arrivé au pouvoir après Gowon, a formé le projet de passer à un programme civil en 1979. Il a mis en place un comité chargé de rédiger la constitution et a réorganisé le pays en 19 Etats. Mais il a été assassiné lors d'un coup d'Etat manqué le 13 février 1976.

Après l’assassinat du général Murtala Mohammed, le général Olusegun Obasanjo est arrivé au pouvoir et a poursuivi le programme de transition vers le régime civil, qui a pris fin en 1979 lorsque le pays est revenu à la démocratie avec le président élu Shehu Shagari. Il faut noter que seulement 50000 membres des forces armées avaient été démobilisés à ce stade (Osaghae 1998: 82). Ainsi, dans la période d'après-guerre, les efforts pour réduire les forces armées sont devenus un thème central des débats sur la réforme militaire et sur la transition du régime militaire vers la démocratie.

La Constitution de 1979 a proscrit les coups d'Etat et écarté les militaires des sphères politiques. Elle a placé le chef d'état-major de la défense sous la responsabilité du président plutôt que sous celle du ministre de la Défense, comme cétait le cas lors de la Ie République. Dans le nouveau système, des comités chargés des affaires de police, de défense et du renseignement ont alors été mis en place à la Chambre des représentants et au Sénat afin de renforcer le contrôle du pouvoir législatif. Cependant, le fait d'avoir placé le chef d'état-major de la défense directement sous l'autorité du président a affaibli ce même contrôle. De plus, les militaires nont pas respecté la nouvelle procédure d'appels d’offres pour les contrats d'Etat ni le protocole d'approbation législative pour l'engagement des dépenses. Cette situation était en partie due au fait que les chefs militaires se sentaient assez puissants pour défier les ordres présidentiels. Par exemple, le major-général Dumuje a ignoré les ordres du président Shagari d'envoyer des troupes en aide aux autorités civiles lors d'une insurrection religieuse à 
Kano. Les militaires ont aussi essayé d'influencer les nominations politiques, notamment en soumettant au président «une liste de leurs candidats préférés » pour les postes ministériels en 1983, juste avant le coup d'Etat du 31 décembre (Alaga \& Akum 2013: 221-222).

Malgré ces difficultés, la guerre civile et les années suivantes ont coïncidé avec un double boom pétrolier qui a permis au Nigéria de développer son industrie de substitution aux importations, d'investir dans les infrastructures et de financer non seulement des importations massives de biens intermédiaires et de biens d'équipement, mais aussi de matières premières et d'autres biens de consommation. Entre 1975 et 1979, l'économie a augmenté de 8,3\% par an et le pays a enregistré un excédent commercial de N2 milliards en 1980, en dépit de la baisse soudaine des prix du pétrole en 1978 (Olukoshi 1993). L’armée s'est développée et a acquis des équipements ainsi que des pièces d'artillerie plus modernes. Elle a également construit des casernes dans tout le pays. Toutefois, laccroissement du réseau de voies urbaines et d'autoroutes ainsi que la mise en place de programmes sociaux ont favorisé la corruption parmi certains militaires de haut rang et leurs collaborateurs. Malgré les efforts déployés sous les gouvernements de Murtala Mohammed et d'Olusegun Obasanjo entre 1975 et 1979 pour instituer des mécanismes budgétaires, un grand nombre d'institutions étatiques comme l'armée n'en faisaient usage que lorsque cela les arrangeait. Ainsi, comme l'ont remarqué Omitoogun et Oduntan, les processus budgétaires et les dépenses dépendaient généralement davantage de la personnalité du chef de l'Etat du moment que des mécanismes institutionnels (Omitoogun \& Oduntan 2006: 158).

C'est dans ce contexte que l'excédent commercial du Nigéria s'est transformé en un déficit de N300 millions dès 1983, et que sa dette extérieure a atteint N21,38 milliards, soit une augmentation ahurissante de 989,2\% depuis 1980 (Adesina 1995: 18). La dette publique intérieure est passée de N4,6 milliards en 1979 à N22,2 milliards en 1983, tandis que le produit national brut a baissé de $8 \%$ en 1982 et de 5,5\% supplémentaires en 1984. L'inflation de $23 \%$ en 1979 est passée à $40 \%$ en 1983. Afin de résoudre la crise grave et soudaine de la balance de paiements, le Nigéria a dû accuser un déficit budgétaire et contracter de gros emprunts auprès d'organismes internationaux privés et officiels de façon à pouvoir financer ses programmes. Ce faisant, le pays a accumulé dénormes dettes. Pour endiguer la crise, le gouvernement Shagari a promulgué une loi de stabilisation économique (dispositions provisoires) en avril 1982, et a de plus introduit plusieurs mesures d'austérité visant à réduire les dépenses du gouvernement ainsi que les importations. Ces mesures ont été renforcées par le gouvernement Buhari, qui avait renversé celui de Shagari lors d'un coup d'Etat militaire en 1983.

Quand Ibrahim Babangida a succédé à Buhari en 1985, il a mis en place un programme de réforme économique en même temps qu'un programme de retour à la démocratie. Or, pendant son mandat, le pouvoir était concentré 
entre les mains du président. Afin de s’assurer des appuis, il a accordé de façon stratégique des promotions, des reconversions, il a nommé des officiers à des postes politiques et a facilité l'attribution de contrats à des officiers à la retraite, en plus d'avoir offert des véhicules à des officiers de certains rangs. A d'autres occasions, il a incité des membres des forces armées à protester contre la société civile lors de manifestations publiques hostiles au gouvernement sous prétexte qu'elles étaient des tentatives pour «détruire la crédibilité de l’armée» ou pour «expulser des militaires hors du gouvernement en les humiliant». Il a déclaré que «seule l'armée pouvait amener les troupes à réintégrer leurs casernes» (Adekanye 1997: 45, 47).

En dépit des ces problèmes, sous le mandat de Babangida, le sort des Igbos dans les rangs de l'armée a commencé à s'améliorer progressivement pour la première fois depuis la guerre civile quand Ebitu Ukiwe, lui-même originaire de cette ethnie, a été brièvement nommé chef détat-major général. Mais la question de l'équilibre régional a continué à se poser au Nigéria malgré un recrutement dans l'armée et dans d'autres services officiels subordonné au principe du caractère fédéral, disposition figurant dans la Constitution de 1979 et exigeant que les nominations à des postes publics reflètent de manière équitable la diversité linguistique, ethnique, religieuse et géographique du pays.

Lannulation par Babangida des élections présidentielles du 12 juin 1993, censée conclure le programme laborieux de transition vers le régime civil, a déclenché une crise politique majeure, causant le mécontentement de la population, creusant encore davantage le fossé entre les différents groupes ethnolinguistiques et encourageant les revendications pour une refonte ou une réforme de l'armée. De ce fait, Babangida sest écarté, laissant un gouvernement provisoire mener à bien la transition vers le régime civil. Cependant, ce gouvernement provisoire a été déclaré illégal par une cour de justice: le général Sani Abacha l'a renversé plus tard en 1993 lors d'un coup d'Etat sans effusion de sang. Il a anéanti toutes les institutions démocratiques existantes et de nouveau tenté de passer à un régime démocratique par le biais d'un programme destiné à faire de lui un président civil. Mais il est mort subitement le 8 juin 1998. Son successeur, le général Abdulsalami Abubakar, a entrepris une campagne pour encourager les militaires à regagner leurs casernes avant le 29 mai 1999.

Le gouvernement du général Abdulsalami Abubakar a entamé son projet de réforme de l’armée avec un programme de transition d'une durée de 10 mois. Le point le plus important était le plan du président de persuader les militaires de réintégrer leur caserne. D’autre part, Abubakar a: entrepris d’améliorer la qualité de vie des soldats, s'engageant à augmenter les salaires des recrues et des officiers avant la fin de 1998; mis sur pied un comité chargé d’organiser une série d’ateliers à l'intention des forces armées et de la police sur le bien-être; la «reprofessionalisation» et l'importance de revenir à la démocratie; apaisé les tensions politiques en relâchant des personnalités de l'opposition et des activistes de la société civile qui avaient défié le régime du général Sani Abacha; 
abrogé plusieurs décrets qui rendaient légales les arrestations arbitraires sans procès et d'autres restrictions des libertés publiques; promis d'examiner plusieurs cas de violation des droits humains. Le général a alors pu s'atteler à l'élaboration d'une constitution conçue selon les principes démocratiques.

Aux termes de la nouvelle constitution, la chaîne de commandement et la conduite des opérations militaires ont été placées sous la responsabilité du président en sa qualité de commandant en chef à titre civil, sous la supervision expresse de l'Assemblée nationale. Dans une large mesure, cela a empêché que l'armée soit directement impliquée dans les processus politiques de la transition, même si elle a continué à influencer indirectement les choix des candidats à la présidence. Cependant, à part ces quelques mesures élémentaires, aucun programme spécifique de réforme à long terme n’a été mis en place. La tâche de refonder le secteur de la sécurité a donc été laissée au gouvernement civil entrant.

\section{La démocratisation du secteur de la sécurité entre 1999 et 2007}

En mai 1999, Olusegun Obasanjo est devenu président d’un régime civil, la IVe République. La réforme militaire est passée en tête de liste des priorités du nouveau gouvernement car elle s'articulait autour de cinq questions essentielles: i) les revendications de la région du Sud concernant la domination du Nord, notamment la prédominance des ethnies du Nord dans l'armée; ii) la crainte que le régime militaire durant 15 des 25 premières années de l'indépendance n'ait installé l'habitude d'utiliser les coups d'Etat pour résoudre les désaccords, risquant ainsi de compromettre la règle démocratique dans l'avenir; iii) le besoin de réorganiser l’armée de façon à la rendre plus resserrée et efficace, y compris en s'attaquant à la corruption interne afin de rétablir une gestion prudente et adéquate des ressources et permettre aux forces d’être mieux préparées; iv) l'importance de renforcer les liens entre civils et militaires qui avaient rendu ces derniers dépendants de dirigeants civils et de démilitariser la société nigériane en s'efforçant d'atténuer sa culture de violence, son aversion pour les débats et l'utilisation de mesures extrêmes dans les conflits internes (Adejumobi 1999); v) la nécessité de traiter plusieurs situations interactives nées du besoin de dépolitiser l'armée en révoquant des soldats occupant des postes politiques de façon à ce que le statut militaire ne confère plus un pouvoir politique ou un privilège financier conséquent. Il s'agissait là d'un point particulièrement important vu la tradition permettant aux cadets de s'enrôler pour accéder plus vite à des postes politiques. Mais, il y avait le risque que des soldats exposés aux privilèges, aux promotions rapides et à l'enrichissement extravagant, qui allaient souvent de pair avec les fonctions politiques au Nigéria, ne se contentent plus de la vie morne et sans faste dans les casernes (Adekson 1979). Il a donc fallu réorienter l'armée vers son rôle initial et inciter les officiers à ne plus avoir de 
velléités d’ordre politique. Un autre problème était le besoin de «reprofessionnaliser» l'armée afin de remédier aux effets indésirables de sa politisation sur l'esprit de corps, le respect de la hiérarchie militaire et la discipline en général. Les échecs économiques et politiques ainsi que les coups et contre coups d'Etat récurrents sous le régime militaire ont décrédibilisé le discours des militaires qui prétendaient être sur la bonne voie. De plus, la corruption endémique, qui avait marqué le régime militaire, a terni l'image de l'armée en tant que gardienne de l'unité et de l'intégrité de l'Etat nigérian.

Peu après avoir pris ses fonctions en 1999, le président Obasanjo a limogé plus d'une centaine de généraux et autres officiers de rangs intermédiaires, placés à des postes politiques. Grâce à cette décision stratégique, l’armée a été purgée d'hommes politiques en uniforme, et un processus global de réforme a pu commencer. Ainsi, sous Obasanjo, l’effort de réforme a été inspiré par le désir de prévenir toute tentative par la région du Nord d'utiliser l'armée pour rétablir son hégémonie politique. Etant donné le nombre disproportionné d’officiers originaires du Nord qui avaient exercé des fonctions politiques sous le régime militaire, l'initiative de "reprofessionnaliser» l'armée en mettant ces officiers à la retraite a contribué à créer un certain équilibre dans la représentation régionale aux niveaux supérieurs de la hiérarchie militaire. A la suite d'une révision des postes d'état-major et d'autres services, de nouveaux officiers supérieurs issus d'horizons plus divers ont été désignés pour remplacer ceux partis à la retraite. En 2010, la nomination d'un Igbo, le général Azubuike Ihejirika, au poste d'état-major a été saluée dans les médias car il était le premier officier de cette ethnie à exercer ces fonctions depuis la fin de la guerre civile en 1970.

Dans le discours qu'il a prononcé au National War College (à présent appelé National Defence College) en 1999, il a présenté les principaux volets du projet de réforme:

1. la nomination d'un président civil élu au poste de commandant en chef des forces armées et la suprématie des fonctionnaires d'Etat élus sur les officiers nommés à tous les niveaux;

2. la direction du ministère de la Défense et d’autres institutions stratégiques par des civils;

3. le fait que les décisions relatives aux objectifs et à la conduite des opérations militaires doivent servir les buts politiques et stratégiques fixés par l'autorité civile;

4. le respect des principes du droit civil lors de toutes les enquêtes et procès militaires;

5. le droit pour les autorités civiles (la Cour suprême) d'examiner toutes les mesures ou décisions prises par des magistrats militaires;

6. l'incorporation d'autres instruments destinés à consolider la suprématie des autorités civiles, par exemple des clauses constitutionnelles ou des modalités de contrôle par le pouvoir législatif (Manea \& Rüland 2013: 65). 
Bien qu'Obasanjo ait par la suite produit toute une série de programmes de réforme couvrant une vaste gamme de thèmes relatifs à la gouvernance, la réforme de l'armée était omise la plupart du temps. En effet, ni la Stratégie nationale pour l'autonomisation et le développement économiques ni le document Vision 20: 2020 (NNPC 2004; 2009), élaborés sous le régime d'Obasanjo, ne mentionnaient la réforme du secteur de la sécurité ou sa gouvernance. En examinant le contenu de la réforme du secteur de la sécurité durant cette période et les efforts déployés, Manea et Rüland (2013: 64) ont constaté qu'il n'y avait pas de concept global ni de volonté politique suffisante pour l'appliquer. Les mesures mises en œuvre par le régime Obasanjo pour réformer l'armée étaient les suivantes:

1. le pouvoir de nommer et de révoquer les chefs de service;

2. la reconversion du ministère de la Défense en une instance civile;

3. la création d'une Commission d'enquête sur les violations des droits humains (la "Commission Oputa») et d'une Commission sur les droits de l'homme;

4. la réforme de la justice militaire stipulant que toutes les décisions du tribunal militaire seraient dorénavant soumises à l'examen de la Cour suprême ;

5. la réforme des relations civilo-militaires (avec la MPRI);

6. le contrôle législatif du budget de la défense le soumettant à l'examen approfondi de l'Assemblée nationale et à l'approbation des organes de supervision tout au long du processus;

7. l'énoncé d'une Stratégie de défense nationale en 2006 (Nigéria 2006).

Cependant, vers la fin du régime Obasanjo, lélan s'est ralenti en raison des querelles permanentes entre le président et le pouvoir législatif, notamment concernant les tentatives d'Obasanjo pour inclure dans la constitution une disposition prévoyant un troisième mandat présidentiel. Ces préoccupations politiques l'ont détourné de son effort de réforme, affaiblissant les chances de voir l'Assemblée nationale promulguer la nouvelle législation relative à l'armée ainsi que les amendements à la constitution en faveur de la réforme militaire.

Comme le remarquent Aiyede (2013:177-179) ainsi que Manea et Rüland (2013: 64-69), les espoirs d'une réforme de l'armée étaient d'autant plus minces qu'il n'y avait pratiquement aucun soutien de la part des médias ni de la société en faveur du contrôle démocratique de l'armée. Plusieurs occasions de réforme ont été gaspillées, y compris celles évoquées lors de la Conférence de 2005 pour la réforme politique. En plus de la création du Conseil national de sécurité pour le renseignement et de la Commission nationale pour les services de sécurité, la conférence recommandait l'adoption d'une disposition constitutionnelle modifiant l'article 1(2) de la Constitution de 1999 afin de se prémunir contre les coups d'Etat. Les autres recommandations étaient: la réorientation politique de l’armée; une nouvelle formation des forces armées afin 
d'améliorer leur professionnalisme; la réorganisation de la société DICON (Defence Industries Corporation of Nigeria); la décision d'investir dans une recherche et un développement axés sur les applications militaires et financés à hauteur d'au moins $5 \%$ du budget de la défense; la création d'un institut de technologie dans l'enceinte de l'Ecole nationale de défense pour proposer des études de $2^{\mathrm{e}}$ et de $3^{\mathrm{e}}$ cycle en génie maritime/aéronautique, en technologie des armements et en informatique; la création d'une école de guerre interarmées; l'amélioration des services sociaux pour le personnel militaire; et l'optimisation de la participation de l'armée dans les opérations de maintien de la paix. Mais, sur les 116 amendements constitutionnels proposés, aucun ne se rapportait aux questions militaires. En fin de compte, cela n'a guère eu d'importance car l'Assemblée nationale s'étant opposée à la proposition d'inclure un troisième mandat présidentiel, toutes les modifications à la constitution ont été rejetées.

Le processus de réforme a également souffert de la réticence des militaires à recourir à l'aide extérieure, en particulier entre 1999 et 2001 lorsque Victor Malu était chef d'état-major. Toutefois, le président Obasanjo a invité la MPRI et l'Equipe britannique de conseil sur la défense à contribuer au programme de réforme. Des officiers de haut rang ont alors décrété que les connaissances de la MPRI en matière de relations entre civils et militaires, dont l'enseignement était la raison de leur venue au Nigéria, étaient déjà inculquées dans les établissements militaires nigérians. De plus, selon eux, le soutien proposé pour «reprofessionnaliser» l'armée nétait pas vraiment nécessaire et n'avait pas fait l'objet d'une discussion préalable avec les dirigeants militaires; en fait, ce soutien était contraire à leurs attentes s'agissant de l'aide extérieure. ${ }^{1}$

Sous le gouvernement Yar' Adua, qui a succédé au président Obasanjo en 2005, les efforts de l'exécutif civil pour poursuivre la réforme de l'armée ont été à nouveau ralentis, quand bien même la sécurité figurait parmi les sept thèmes inscrits à l'ordre du jour de ce gouvernement. Ceci était surtout dû à la maladie et, pour finir, au décès du président, incapable d’exercer ses fonctions pendant la majeure partie de son mandat entre 2007 et 2010. La présidence était assurée par son entourage, une coterie dont sa femme faisait partie. Lorsque le président s'est rendu en Arabie saoudite, il n’a pas confié officiellement ses pouvoirs au vice-président Goodluck Jonathan pendant la durée de son voyage, comme il est stipulé dans la constitution; au lieu de cela, son état de santé a été gardé secret par ses proches. On a réellement craint que les militaires ne s'emparent du pouvoir en mars 2010 quand le président est subrepticement revenu à l'aéroport d’Abuja de nuit sans en informer le vice-président, qui lui a succédé en fin de compte plus tard cette année-là (Adeniyi 2011: 237).

Le président Goodluck Jonathan a ensuite remporté les élections de 2011. Cependant, aucune initiative importante sur la question de la réforme de l'armée ne figurait au programme de transformation de son gouvernement, malgré l'aggravation de la menace de Boko Haram sous son mandat. Les dirigeants 
militaires changeaient fréquemment, preuve que leurs nominations et mises à la retraite étaient contrôlées par des civils; ce qui n’a pas empêché le budget de la défense d'augmenter considérablement pendant cette même période. Ces décisions sont maintenant subordonnées au contrôle parlementaire. En ce qui concerne la nomination des chefs deétat-major des trois armées, le président a commencé à solliciter l'approbation du Sénat en 2013 après la décision du juge Adamu Bello le 1er juillet 2013 de rendre cette mesure obligatoire. Avant cette date, le président nommait et révoquait les chefs d'état-major sans consulter le Parlement. A l'heure actuelle, le président nomme les chefs d'état-major, puis soumet la liste au Sénat pour confirmation. A ce jour, aucune de ces nominations ou mise à la retraite n'a été rejetée.

\section{Pour un changement durable}

Malgré une réforme en apparence ralentie et une opposition de l'armée à l'implication d'intervenants extérieurs, les dirigeants militaires ont élaboré et mis en œuvre leur propre programme de réforme. Toutefois, les mesures dépendaient des priorités de chacun des chefs d'état-major de l'armée de terre qui se sont succédés. Par exemple, en mai 2004 le chef d’état-major de l'armée de terre à l'époque, le général Martin Luther Agwai, a établi un Comité de la gestion du changement chargé d'évaluer les besoins en matière d'installations, d'équipements et de formation de l'armée de terre nigériane et de faire face aux menaces et défis à relever pour les dix prochaines années et au-delà. Un plan cadre pour la transformation de l'armée (Framework for the Transformation of the Nigerian Army in the Next Decade - Volumes $1 \& 2$ ) a été rédigé par la suite et le bureau de transformation de l'armée Nigeriane a été créé en 2006 pour conceptualiser, élaborer et mettre en application des plans à court, moyen et long terme.

En 2006, lorsqu'Agwai est devenu chef d'état-major de la défense (CEMD), il a essayé de propager ces idées dans les autres services de l'armée. Il a établi le Comité de transformation des forces armées au sein du ministère de la Défense, faisant ainsi un premier pas vers la transformation de l'armée dans son ensemble. En 2008, le comité a rédigé un document stratégique, deux volumes d'une doctrine commune aux forces armées et un plan d'organisation pour la haute direction de la défense (MOD 2008a; 2008b). Il y était prévu de réviser la stratégie militaire tous les cinq ans et la doctrine tous les deux ans. Cependant, à ce jour, la structure de gestion envisagée n’a pas était mise en place et aucune révision n'a eu lieu. Le comité a d'autre part publié des documents concernant ses projets pour:

1. réorganiser le ministère de la Défense afin d'améliorer la gestion de la défense nationale;

2. définir une stratégie militaire nationale ainsi qu'une doctrine opérationnelle commune; 
3. fournir des moyens pour renforcer les compétences et la motivation des personnels;

4. mettre en place des processus pour l'achat et l'entretien en commun d'équipements militaires majeurs et accessoires;

5. créer une capacité de recherche et développement et de services industriels dédiés à la défense; et

6. mettre en place un dispositif militaire fiable capable de relever les défis actuels et à venir.

Parmi tous ces documents, il faut souligner la Politique nationale de défense de 2006 car elle a été élaborée pratiquement sans l'aide du Parlement ou de la société civile (Aiyede 2013:176). Cette politique a bien adopté un concept d'opérations interarmées pour améliorer l'efficacité opérationnelle de l'armée, mais son application s'est trouvée compromise par l'absence d'un contrôle centralisé. Cette difficulté était due aux ambigüités contenues dans les articles de la loi de 2004 sur les forces armées nigérianes, lesquels attribuaient la responsabilité des opérations au CEMD, ainsi que dans l'article 217 de la Constitution de 1999, dans lequel les pouvoirs du chef deétat-major de la défense nétaient pas évoqués puisque seuls les chefs d'état-major de chaque armée étaient mentionnés et que ceux-ci prennent leurs ordres directement auprès du ministre de la Défense. Certes, ce système réduit le rôle du CEMD à celui d'un simple conseiller auprès du commandant en chef chargé, lui, de diriger les opérations des forces armées même si le CEMD est en théorie responsable de la coordination et de l'intégration des activités des trois armées. De plus, le CEMD est habituellement choisi parmi les chefs détat-major de chaque armée et cumule les deux fonctions dès sa nomination. Ce système suppose que pour être bien organisées et efficaces au combat, les forces armées doivent être dirigées par un CEMD investi de pouvoirs qui l'habilitent à décider des activités des trois armées, à les superviser et en assurer la coordination. Ces prérogatives pourraient néanmoins restreindre l'influence du président sur les dirigeants militaires: un système qui place le président ou le ministre de la Défense au-dessus du CEMD et des chefs des trois armées permet de cultiver la loyauté, d'accorder des distinctions et par conséquent d'exercer une influence certaine sur les forces armées. Ces contradictions proviennent du fait que la loi de 2014 sur les forces armées nigérianes a été promulguée afin de préserver le contrôle présidentiel sur la hiérarchie militaire. Le fait chefs d'état-major entrent en compétition pour être reçus par le président ou le ministre de la Défense a été perçu comme une garantie contre les coups d'Etat ou l'éventualité que les militaires s'entendent entre eux pour faire valoir des revendications auprès des dirigeants politiques. Mais d'un autre point de vue, retirer des pouvoirs au CEMD, notamment ceux relatifs au budget et aux opérations sur le terrain, pour les donner à chaque armée permet aux chefs détat-majors concernés dêtre plus autonomes et de décider eux-mêmes de leur budget; pouvoirs qu'ils sont très réticents à abandonner. Dans la pratique, comme le remarquent Menea et Ruland, le résultat 
est que «les achats des équipements militaires sont en grande partie contrôlés par les chefs de chaque armée et dans une moindre mesure par le CEMD et le ministre de la Défense (Manea \& Rüland 2013: 73). D’autre part, il est de coutume que le président finance les équipements militaires au moyen de décrets libérant des ressources hors budget. C'est pourquoi le président tout comme les chefs des services sont intéressés à maintenir le statu quo.

Le fait que le processus d'acquisition soit empreint de corruption à cause de ces contradictions, explique pourquoi celles-ci restent bien ancrées et sont loin dêtre réglées. ${ }^{2}$ Comme le note Oyegbile (2014), un processus d'acquisition en proie à la corruption est, en partie du moins, responsable du déficit en équipements et du mauvais moral des troupes. Il cite un officier supérieur à la retraite montrant à quel point cette situation est mal vécue parmi les officiers de haut rang:

Dans ce pays, la guerre contre le terrorisme ou même une guerre conventionnelle ne peut être gagnée dans les conditions actuelles. Notre système de financement de l'armée est profondément corrompu et permet les abus les plus flagrants. Les budgets militaires ne devraient jamais être confiés à des chefs militaires ni au chef d'état-major de l'armée de terre comme c'est le cas actuellement (Oyegbile 2014).

La doctrine commune inscrite dans la Politique nationale de défense mettait l'accent sur la primauté des dirigeants politiques, des représentants élus et de leurs subalternes dans l'élaboration des politiques et des procédures nationales générales en matière de défense. Parallèlement, elle prévoyait que les militaires donnent leur avis et formulent des recommandations concernant certaines questions professionnelles comme la capacité opérationnelle, les limitations et les prévisions militaires. Elle prévoyait aussi que les dirigeants restent à l'écoute du public et tiennent les citoyens informés au fur et à mesure des progrès de leurs missions, surtout quand il était fait usage de la force (MOD 2008b). Si l'armée est la mieux placée pour fournir des informations précises, complètes et en temps utile afin de permettre à l'Assemblée nationale de remplir correctement ses obligations constitutionnelles concernant les questions militaires, les dirigeants civils ont tout de même choisi de ne pas appliquer pleinement la doctrine commune comme prévu. Par exemple, depuis 1996, les présidents civils ont nommé et mis à la retraite les membres de la haute direction des forces armées conformément aux dispositions de la constitution, mais ils ont agi sans l'aval du législatif exigé par la loi. Ainsi, Festus Keyamo, un militant des droits humains et avocat de profession, a obtenu un jugement de la Haute cour fédérale en 2013, qui a contesté la pratique de nommer des chefs d'état-major sans l'approbation de l'Assemblée nationale. La cour a déclaré les nominations antérieures nulles et non avenues. C'est pourquoi le président Jonathan a demandé au Sénat de confirmer les nominations qu'il a annoncées le 16 janvier 2014, 
ce qui a été fait concernant les nouveaux chefs d'état-major le 29 du même mois (Ojiabor 2014: 6). A partir de ce moment-là, la société civile a surveillé les abus de l'exécutif et a pu utiliser le système judiciaire pour les dénoncer. Malheureusement, le cas est resté isolé.

En fin de compte, après le régime Obasanjo, les efforts investis pour promouvoir le programme de transformation ont capoté par manque d’engagement. En conséquence un nombre restreint de mesures de réforme ont été élaborées et mises en œuvre par les chefs de chaque armée en vue de servir leurs propres intérêts. Par exemple, à l'époque du CEMD Paul Dike, également maréchal en chef de l'armée de l'air, plusieurs programmes, séminaires, visites, conférences et ateliers ont été organisés pour expliquer et faire avancer le processus de changement. Le bureau chargé de gérer la transformation au quartier général de la défense a puisé dans les ressources de groupes de réflexion indépendants et d'organisations privées pour sensibiliser les militaires à l'importance d'entretenir de bonnes relations avec les médias et la société civile (Onwudiwe \& Osaghae 2010). Les trois armées ont également mis en place des bureaux de relations publiques et l'armée de terre a créé un réseau étendu (le Wide Area Network Infrastructure) pour faciliter l'accès à l'information et améliorer la performance, les compétences et l'efficacité opérationnelle (Bojie 2011:3).

Au sein de l'armée de terre nigériane, des conférences, séminaires et ateliers de sensibilisation ont eu lieu, et les livres et manuels ont été revus afin de renforcer les connaissances des personnels concernant le processus de transformation. Ces séminaires et ateliers se sont tenus à Abuja et dans tous les quartiers généraux. Ils avaient pour thème par exemple: la coopération entre civils et militaires, le système d'état-major continental ou les changements de comportement. Les soldats ont reçu un nouvel uniforme donnant d'eux une image plus avenante. De même, en 2011, le général Onyeabor Azubuike Ihejirika, alors chef d'état-major de l'armée de terre, a créé le Bureau des affaires civilo-militaires pour améliorer l'image de l'armée, gagner le soutien du public et traiter les questions relatives aux droits humains, à l'Etat de droit, aux négociations, aux communications et à la gestion des conflits (Alaga \& Akum 2013: 229).

Juste au moment où la réforme militaire semblait avoir démarré, les difficultés sécuritaires du Nigéria se sont aggravées. Les enlèvements et les cambriolages ainsi que les actes de terrorisme, pour la plupart perpétrés par Boko Haram, ont amené les agences de sécurité à se réorienter. A présent, presque toutes sont engagées dans la lutte contre ces troubles. Par exemple, l'armée, la police et d'autres agences de sécurité ont mis en place des cellules antiterroristes. De plus, les militaires se sont progressivement engagés dans d'importants programmes de maintien de l'ordre dans les 28 Etats du pays, ce qui signifie qu'ils sont souvent trop peu nombreux. L'intervention de l'armée dans des zones communales en conflit, la lutte contre la criminalité et le terrorisme ont catalysé mais aussi engendré des polémiques (Falana 2014). Selon l'organisation Crisis Group, le Nigéria a utilisé trois moyens pour faire face aux difficultés liées à la sécurité: 
les augmentations budgétaires, le renforcement de la législation antiterroriste et l'accroissement de la capacité militaire.

Ces dernières années, les efforts contre le terrorisme ont été élargis de façon à couvrir le secteur non gouvernemental de la sécurité, notamment la défense civile, les entreprises de sécurité privées, la collaboration entre citoyens et agences de sécurité, et le système judiciaire. La loi sur la prévention du terrorisme a été promulguée en 2011. Elle a été suivie en 2012 d'un amendement chargeant le Bureau du conseiller pour la sécurité nationale de coordonner l'action antiterroriste à l'échelle du pays. Cette mesure a été prise afin de prévenir les rivalités entre les agences de sécurité pour déterminer qui devrait diriger les opérations conjointes. Le gouvernement s'est également efforcé d’améliorer la formation, la gestion du personnel, les équipements (notamment pour les combats rapprochés) et la coordination. Par exemple, plus de 7000 agents de sécurité issus de l'armée, de la police et du corps de sécurité et de protection civile nigérian ont été formés aux différentes techniques utilisées en combat urbain, la lutte contre le terrorisme et contre les insurrections, le renseignement, les opérations amphibies, l'ouverture de brèches par la démolition ou l'emploi d'explosifs, et les moyens de transmission tactiques. Le Centre de lutte contre le terrorisme et contre les insurrections, institution d'instruction militaire spécialisée située à Jaji dans l'Etat de Kaduna, a formé des élèves issus des forces armées et de la police.

Cependant, comme l'a fait remarquer Leren Blachard du Service de recherche du Congrès sur les affaires africaines, les gouvernements qui se sont succédé au Nigéria ont mis du temps pour permettre aux services de sécurité de participer aux programmes de formation américains. Décrivant le Nigéria comme un partenaire avec lequel il est «extrêmement difficile» de collaborer, elle a constaté que les troupes étaient «lentes à s'adapter aux nouvelles stratégies, aux nouvelles doctrines et aux nouvelles tactiques» (Akinloye 2014: 1). Néanmoins, après les enlèvements de Chibok, le président Jonathan a fait appel à l'aide extérieure. La Chine, les Etats-Unis, Israël, la France et le Royaume-Uni ont promis leur soutien, et la garde nationale californienne est actuellement en train de mettre sur pied le $143^{\mathrm{e}}$ bataillon d'infanterie, force toute spécialement formée pour affronter Boko Haram dans son bastion rural (Iroegbu \& Adinoyi 2014: 1).

En réponse au conflit sans fin dans le delta du Niger et à la vague d'actes criminels (surtout des enlèvements) dans le Sud-Est du pays, le président a rouvert les casernes d'Ahiara qui avaient été fermées en 1992 lors de la réorganisation de l'armée. Les baraquements abritent la toute nouvelle $14^{\mathrm{e}}$ brigade ainsi que sa garnison, un bataillon et d'autres unités de soutien. Il a été proposé d'installer un $145^{\mathrm{e}}$ bataillon à Ikot Umoh Essien dans l'Etat d'Akwa-Ibom, un $144^{\mathrm{e}}$ bataillon à Umuna dans l'Etat de Rivers et un régiment d'artillerie dans l'Etat d'Ebonyi (Onuorah 2011:4).

Le gouvernement a augmenté le budget de la défense de N100 milliards (US\$625 millions) en 2010 à N927 milliards (US\$6 milliards) en 2011, et à N1 
trillion (\$6,25 milliards) en 2012, 2013 et 2014. Bien que secteur de la défense se soit vu attribuer un tiers du budget du gouvernement fédéral en 2014, en août de cette même année, le président Jonathan a soumis à l'Assemblée nationale une demande de prêt de \$1 milliard pour pouvoir rééquiper l'armée, ce qui lui permettrait de lutter contre les insurrections et de faire face aux autres défis sécuritaires. Plus de 30 chars blindés et deux hélicoptères de combat équipés de systèmes de vision nocturne ont été acquis récemment et déployés contre les rebelles dans le Nord-Est, tandis que l'armée de l'air a pris livraison de six avions à réaction (McGregor 2015).

Létat d'urgence a été d’abord déclaré dans les Etats du Nord-Est le 13 mai 2013 en raison de l'intensification de l'activité de Boko Haram. Trois prolongations ont été automatiquement approuvées par le Parlement dans le cadre de ses fonctions de contrôle. Le 17 novembre 2014, le Conseil national de défense a décidé de prolonger une quatrième fois l'état d'urgence dans les Etats d'Adamawa, d'Yobe et de Borno. Mais la Chambre des représentants a rejeté la demande lorsqu'elle a été présentée au Parlement pour approbation. Le Sénat, qui était divisé sur la question, n'est pas intervenu avant l'expiration de l'état d'urgence. La plupart des membres du Parlement pensaient qu'il n'avait eu aucun effet sur les rébellions et que le président devrait recourir aux dispositions contenues dans la constitution et dans l'article 8 de la loi sur les forces armées pour se conférer le droit d'envoyer les troupes dans les régions du pays en proie à des troubles (Adejuwon 2014). Létat d'urgence avait été approuvé auparavant parce que les militaires avaient expliqué au Parlement que sa mise en œuvre était nécessaire eu égard au manque de visibilité et à la menace pour la paix dans les territoires en question: la mesure faciliterait le travail des services de renseignement ainsi que l'action contre les terroristes de Boko Haram et elle permettrait aux personnels de l'aide militaire étrangère de pénétrer au Nigéria en toute légalité.

Ainsi, la lutte contre le terrorisme a pris une importance primordiale dans les activités du secteur de la sécurité, surtout depuis l'enlèvement de plus de 200 jeunes filles à Chibok en avril 2014 et l'intensification des agissements de Boko Haram, qui s'est ensuivie. Ces événements font ressortir les insuffisances et les déboires du programme de réforme du secteur de la sécurité. D’autre part, l'action antiterroriste a suscité l'intérêt et le soutien de la communauté internationale, même s'il a fallu pour cela soulever le voile sur les faiblesses de l'armée devant les médias et le public. Dans leurs déclarations, des gouvernements étrangers intéressés à apporter leur assistance et à travailler avec l'armée ont émis des critiques concernant sa capacité et son état de préparation. Par exemple, Alice Friend, directrice principale du Pentagone pour les affaires africaines, a déclaré que «l'armée nigériane avait affaire aux mêmes problèmes de corruption que toutes les autres institutions du pays. La majorité des fonds accordés à l'armée est siphonnée par le haut.» (Schmitt \& Knowlton 2014). D’après les médias, qui suivent de près la situation avec les rebelles, les efforts 
déployés ces derniers temps pour faire avancer la réforme se sont révélés au mieux inefficaces et inachevés; plusieurs améliorations ont même vu leur cours s'inverser.

La corruption s'est révélée particulièrement préoccupante ces derniers temps. Par exemple, selon Nossiter (2014), un journaliste au New York Times, certains diplomates étrangers étaient d’avis que l’armée nigériane avait involontairement ralenti la traque des ravisseurs des jeunes filles de Chibok par leur inefficacité et leur incapacité:

Larmée est si mal entraînée, si piètrement équipée et si gangrenée par la corruption, quelle est incapable de retrouver les jeunes filles. Et, en plus, elle est en train de perdre le combat plus vaste contre Boko Haram. Le groupe de terroristes a déjà pris le contrôle d'une grande partie du Nord-Est du pays, tandis que les troupes se retirent des sites vulnérables pour éviter l'affrontement et sécartent de leur chemin, même lorsqu'ils massacrent des civils (Nossiter 2014).

Les médias nigérians ont évoqué les mêmes faits. Pendant ce temps, en février 2014, Kashim Shettima, gouverneur de l'Etat de Borno, a déclaré que les combattants de Boko Haram étaient mieux armés et davantage motivés que les troupes du gouvernement. En mai 2014, des soldats des unités militaires les plus importantes chargées d'affronter Boko Haram, en colère d'avoir dû subir des pertes dans leurs rangs suite à une embuscade, ont ouvert le feu sur le véhicule transportant leur propre commandant, le major-général Ahmadu Mohammed. Dans la presse, les relations entre les militaires et les médias ont pris le pas sur la lutte contre Boko Haram. Le climat de méfiance qui en a résulté est, en partie du moins, imputable à la mauvaise gestion de l'information de la part de l'armée.

La persistance et l'intensité avec lesquelles l'armée a été critiquée pour ses interventions contre Boko Haram ont conduit le major-général Chris Olukolade, porte-parole de l'armée, à demander aux médias d’arrêter de saper le moral des officiers et des soldats au front (Agbambu 2014: 8). Mais à cause de la mauvaise gestion de l'information, les médias ont commencé à faire circuler de folles spéculations selon lesquelles certains officiers supérieurs auraient pu agir de connivence avec Boko Haram et lui avoir fourni du matériel et des fonds. Par exemple, Stephen Davies, l'Australien nommé par le gouvernement nigérian pour aider à négocier la libération des jeunes filles kidnappées, a accusé un ancien chef détat-major de l'armée de terre dêtre l'un des «parrains» de Boko Haram. Il y a aussi eu des rumeurs selon lesquelles les informations fournies par des officiers auraient aidé les rebelles à monter des embuscades contre des convois militaires (par ex. celle qui a conduit à la mutinerie de mai 2014 contre le major-général Ahmadu Mohammed) et à mener des attaques contre des baraquements et des postes avancés dans le fief de 
Boko Haram au nord-est du pays. En juin 2014, la presse locale a publié plusieurs articles sur des officiers supérieurs jugés en cour martiale pour avoir fourni des armes et des renseignements à des extrémistes de Boko Haram. En réponse à cela, l’armée a confisqué les journaux dans les kiosques pour éviter qu'ils ne circulent dans les villes alentour.

Cependant, en août 2014, les médias ont exprimé violemment leurs inquiétudes concernant la force et la capacité de l'armée lorsque Boko Haram s'est emparé de Gwoza, une petite bourgade dans le Sud de l'Etat de Borno; les rebelles avaient hissé un drapeau et proclamé le califat. L'incapacité de l'armée à déloger rapidement les rebelles ainsi que la bataille qui a suivi entre Bama and Maiduguri ont alimenté les doutes sur les capacités de l’armée. La situation sest encore davantage aggravée quand environ 480 soldats nigérians sont passés au Cameroun après une bataille acharnée avec les partisans de Boko Haram. D’après les médias, cet incident était une désertion, tandis que d’après l'armée il s'agissait d'une manœuvre tactique (Onuorah 2014: 4; Akinlotan 2014: 80). En outre, l'armée a été fortement critiquée pour ne pas avoir respecté les droits de l'homme. Par exemple, dans ses rapports, Amnesty International a à plusieurs reprises accusé l'armée nigériane d'avoir employé la torture et d’avoir procédé à des exécutions sommaires (Amnesty International 2012; 2014).

C'est dans ce contexte que la presse a cessé de recommander au gouvernement de revoir sa stratégie militaire et a commencé à exiger un remaniement complet. Lors de la 58e Semaine de la marine nigériane en mai 2014, le ministre de la Défense, le sénateur Musiliu Obanikoro, a proposé au président Jonathan dorganiser un séminaire présidentiel où les parties prenantes, le Parlement, le secteur privé et d’autres intervenants concernés pourraient se rencontrer afin de «mettre au point une feuille de route pour la renaissance de l'armée nigériane». (Eghaghe 2014: 1).

\section{Conclusion}

Le gouvernement du président Olusegun Obasanjo a joué un rôle primordial dans la réforme du secteur de la sécurité au lendemain de l'indépendance, notamment en ce qui concerne les questions militaires. Même si le programme présenté était vaste, la réforme était surtout axée sur l’exécutif et ne faisait pas grande mention du législatif ni de la société civile. De plus, les tentatives du président pour modifier la constitution afin d'intégrer un troisième mandat présidentiel ont annihilé une grande partie de l'élan et des progrès accomplis jusqu'alors, ce qui a stoppé net les efforts pour amender la législation, lesquels auraient pourtant été bien utiles. Après Obasanjo, les présidents suivants Yar' Adua et Jonathan se sont peu préoccupés de la réforme du secteur de la sécurité, bien qu'ils aient dû faire face à de graves crises sécuritaires. S'ils ont continué à exercer leurs pouvoirs présidentiels pour nommer et mettre à la retraite les 
dirigeants de l'armée, tous deux ont la plupart du temps laissé les forces armées poursuivre le processus de réforme à leur guise. Les réformes ultérieures ont ainsi reflété les convictions des dirigeants alors en place, qui étaient davantage axées sur les améliorations techniques et l'efficacité plutôt que sur la gouvernance démocratique per se.

Le programme de réforme a bénéficié d'un élan considérable sous le commandement du lieutenant-général Luther Agwai, qui a présenté le concept de la transformation à l'armée à l'époque où il était chef d'état-major de l'armée de terre, et il a employé cette expression dans toutes les armées lorsqu'il est devenu CEMD. Cependant, son successeur le lieutenant-général Azazi, nétait pas d'accord avec cette façon de voir les choses: certes, en tant que conseiller en chef pour la sécurité nationale auprès du président, il a plus tard déclaré que:

Depuis le début, l'idée était de dépasser le stade de l'opacité et d'accepter la participation du public car, en fin de compte, toute stratégie de sécurité devrait prendre en compte les objectifs du pays et choisir la meilleure approche pour progresser sans obstacles (Oloja \& Onuorah 2011:1).

La réforme de l'armée a connu un renouveau lorsque le chef de l’armée de l'air Paul Dike est devenu CEMD dans le cadre d'un programme de réforme axé sur l'amélioration des relations entre civils et militaires grâce à un projet visant à élargir, compléter et renforcer les stratégies de relations publiques existantes. La réforme est depuis au point mort. Aujourd'hui, il reste beaucoup de questions non résolues, notamment les problèmes soulevés par la lutte contre les rebelles dans le Nord-Est. On constate aussi des désaccords parmi les officiers de haut rang, non seulement à propos des meilleures méthodes à adopter pour mener à bien la réforme, mais également à propos du contenu-même de la réforme et du processus de transformation. En effet, certains sont d'avis que le stade des efforts est à présent dépassé et que ce qu'il convient de faire désormais est de renouveler régulièrement les équipements et d'assurer une formation continue de façon à pouvoir répondre aux défis qui se présentent. ${ }^{3}$ Le fait que l'armée se soit montrée méfiante, prudente, voire hostile à l'idée de solliciter une aide extérieure a limité encore davantage les progrès passés et à venir vers un véritable changement.

Comme le font remarquer Aiyede (2013:176) ainsi que Manea et Rüland (2013: 67-69), depuis 1999, le Parlement n’a pas été à la hauteur des attentes au vu de ses importants pouvoirs officiels législatifs, s'agissant également de la création de fonctions de contrôle de la défense et de la sécurité. C’est en partie parce qu'il n’a ni les compétences, ni la volonté politique, ni la confiance nécessaires pour intervenir lorsqu'il est question de la sécurité. Ainsi, son rôle a été limité à la supervision de la défense, y compris la confirmation de nouvelles nominations au poste de CEMD et l'approbation de plusieurs demandes émanant du 
président Jonathan en vue d'élargir l'état d'urgence aux trois Etats du Nord-Est, qui sont le théâtre de la lutte contre les rebelles.

La presse a commencé à s'intéresser de plus près à la sécurité quand les échecs des interventions contre Boko Haram ont attiré l'attention du public. Pourtant, il n'y a encore jamais eu de discussion approfondie au sein des médias et du public sur le secteur de la sécurité au Nigéria. La participation de la société civile au processus de réforme de l'armée est elle aussi relativement faible. Même si quelques rares organisations ont joué un rôle dans la réforme de la police, il manque les compétences requises pour promouvoir le contrôle démocratique de l'armée dans les médias et la société civile. Cela explique pourquoi la contribution du législatif, des médias et de la société civile est si médiocre, laissant les instances supérieures de la défense mener les débats. La conséquence directe de toutes ces carences est que le processus de réforme stagne.

Il y a tout de même eu quelques modestes résultats, par exemple l'établissement d'un contrôle civil des forces armées et le limogeage des militaires qui avaient occupé des postes politiques. Bien qu'il reste limité, le contrôle parlementaire sur les questions de défense s'est amélioré. Un programme de réforme exhaustif a été conçu, sous l’égide du Bureau de transformation de l'armée Nigeriane puis du Comité de transformation des forces armées, en vue de réorganiser au mieux la gestion de l'armée, raviver la discipline et l'esprit de corps, moderniser la recherche et le développement, renouveler les équipements et dispenser aux hommes et aux officiers une formation adaptée.

Mais ce programme n'a pas été appliqué avec efficacité et méthode, surtout à cause du manque de volonté tant de la part du Parlement que des dirigeants politiques et militaires. D'autres obstacles sont venus s'ajouter tels que la corruption et de graves défis sécuritaires, notamment les conflits internes et les actes de terrorisme.

Ces dernières années, les agissements de Boko Haram ont le plus contribué à modifier le profil stratégique de la structure organisationnelle de l'armée. La lutte contre le terrorisme a mis en lumière les insuffisances de la réforme du secteur de la sécurité au Nigéria et révélé les faiblesses de l’armée au public, en particulier à l'occasion de l’enlèvement de plus de 200 écolières à Chibok à un moment où l'état d'urgence avait été décrété dans le pays. Des cas de mutinerie et de désobéissance aux ordres, des manifestations organisées par des épouses de soldats exigeant que leurs maris soient mieux équipés et armés pour aller au combat, la trahison de certains officiers et des accusations de corruption sont devenus des sujets d'intérêt public et ont mis la question de la réforme du secteur de la sécurité sur le devant de la scène, en attendant qu'elle soit au cœur de tous les débats publics.

Lenseignement le plus important à tirer de la situation présente et passée au Nigéria est que la réforme du secteur de la sécurité doit s'inscrire dans le contexte plus large de la gouvernance, notamment en insistant sur: l'engagement des dirigeants politiques, les relations entre les départements militaires 
et civils au sein du ministère de la Défense, la motivation du Parlement et de la société civile à traiter les questions de sécurité et leur capacité à le faire, et enfin les principes fondamentaux qui sont le moteur de la politique. L'enthousiasme pour la réforme du secteur de la sécurité, qui a accompagné le processus de démocratisation, sest éteint assez vite et la promesse initiale de rendre l'armée autonome s'agissant de ses décisions et de son avenir n’a pas pu être tenue. $\mathrm{Du}$ fait de l'implication de larmée dans des conflits internes et la lutte contre le terrorisme, les changements survenus au niveau des opérations et de l'organisation militaires, qui se voulaient systématiques, sont devenus désordonnés. Il faut revoir les documents de 2008 relatifs à la réforme de façon à prendre véritablement ces changements en compte s'ils doivent être utilisés pour rajeunir l'armée, réorganiser la haute direction de la défense. L’objectif est de récolter les fruits de la doctrine commune et de la coopération entre les agences et d'établir un cadre pour utiliser efficacement l'aide extérieure.

Etant donné sa situation, le Nigéria doit renforcer ses capacités pour disposer d'effectifs adéquats lui permettant de faire face aux menaces qui pèsent sur la sécurité. Il doit également réorganiser le processus d' acquisition d'équipements afin de loptimiser, réduire la corruption et améliorer les conditions de travail des officiers comme des simples soldats. Tout ce qui se passe avec Boko Haram et d'autres factions rebelles en ce moment montre que le pays doit aussi affiner son réseau et son savoir-faire en matière de renseignement afin de devancer les terroristes et les arrêter avant qu'ils n'aient le temps de frapper. Avant tout, la réforme doit s'inscrire dans un contexte plus large concernant la renégociation des pouvoirs requis pour instaurer un système de gouvernance ouvert à tous et soutenu par un Parlement et des institutions civiles solides, sans oublier l'engagement des médias pour encourager les débats en tenant la population informée afin de lui permettre de se faire sa propre opinion. Ainsi, les principes démocratiques s'en trouveraient encore davantage ancrés dans toutes les activités de la société nigériane, ce qui est une condition préalable essentielle à la réforme du secteur de la sécurité.

\section{Notes}

${ }^{1}$ Entretien avec l'auteur en 2014.

${ }^{2}$ Entretien entre des officiers de l'armée et l'auteur, février 2014.

${ }^{3}$ Discussion entre des officiers de l'armée et l'auteur, février 2014. 